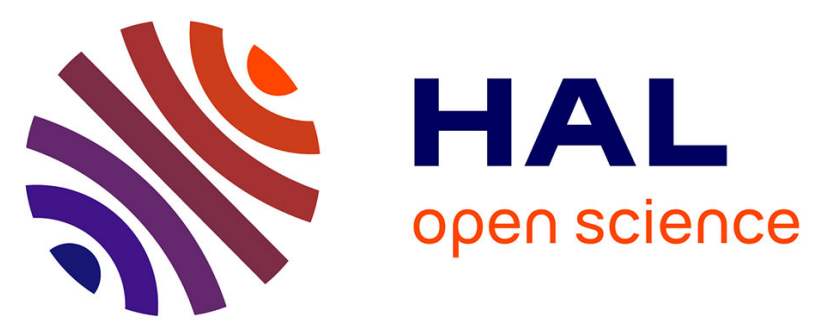

\title{
A tomography diagnostic in the visible spectrum to investigate turbulence and coherent modes in the linear plasma column Mistral
}

Pierre David, Alexandre Escarguel, Yann Camenen, Romain Baude, Didier Ferrand

\section{To cite this version:}

Pierre David, Alexandre Escarguel, Yann Camenen, Romain Baude, Didier Ferrand. A tomography diagnostic in the visible spectrum to investigate turbulence and coherent modes in the linear plasma column Mistral. 2017. hal-01570062

\section{HAL Id: hal-01570062 https://hal.science/hal-01570062}

Preprint submitted on 28 Jul 2017

HAL is a multi-disciplinary open access archive for the deposit and dissemination of scientific research documents, whether they are published or not. The documents may come from teaching and research institutions in France or abroad, or from public or private research centers.
L'archive ouverte pluridisciplinaire HAL, est destinée au dépôt et à la diffusion de documents scientifiques de niveau recherche, publiés ou non, émanant des établissements d'enseignement et de recherche français ou étrangers, des laboratoires publics ou privés. 


\title{
A tomography diagnostic in the visible spectrum to investigate turbulence and coherent modes in the linear plasma column Mistral
}

\author{
P. David, ${ }^{1, \text { a) }}$ A. Escarguel, ${ }^{1, \text { b) }}$ Y. Camenen, ${ }^{1}$ R. Baude, ${ }^{1}$ and D. Ferrand ${ }^{1}$ \\ Aix-Marseille Université, CNRS, PIIM UMR7345, Marseille, France
}

(Dated: 28 July 2017)

In this paper we describe a newly installed tomography diagnostic on the linear magnetized plasma device Mistral. The diagnostic is composed of 128 lines of sight covering a $20 \mathrm{~cm}$ diameter section, and an acquisition rate up to $1 \mathrm{MHz}$. The measurements are done in the visible spectrum using silicon photomultiplier arrays that require lower amplification voltage than photomultiplier tubes for similar gains, and have a fast response. Tomographic reconstruction of a rotating plasma mode is shown to illustrate the capabilities of the diagnostic.

\section{INTRODUCTION}

The linear magnetized plasma device Mistral $^{1}$ presented in Fig. 1 is used for the fundamental study of transport in cold plasmas $\left(T_{\mathrm{e}} \sim 3 \mathrm{eV}\right.$ and $\left.T_{\mathrm{i}} \sim 300 \mathrm{~K}\right) .{ }^{2-7}$. An Argon plasma is created by a thermoionic discharge, with a typical pressure $P \simeq 10^{-2} \mathrm{~Pa}$ and electron density $n_{\mathrm{e}} \simeq 10^{-15} \mathrm{~m}^{-3}$. The Mistral's linear chamber is a $1.2 \mathrm{~m}$ long and $0.4 \mathrm{~m}$ wide cylinder with an axial magnetic field of $0.016 \mathrm{~T}$. The plasma is limited by a grid on each end of the column and an outer cylinder at $r=10 \mathrm{~cm}$. The two grids and the cylinder can be independently polarized between -60 and $60 \mathrm{~V}$. The polarisation of these elements, as well as the pressure, the magnetic field, and the discharge parameters, are used as control parameter to trigger steady global rotating modes, routinely studied on many fundamental or applied devices because of their role in Hall effect thrusters, ion sources or magnetic

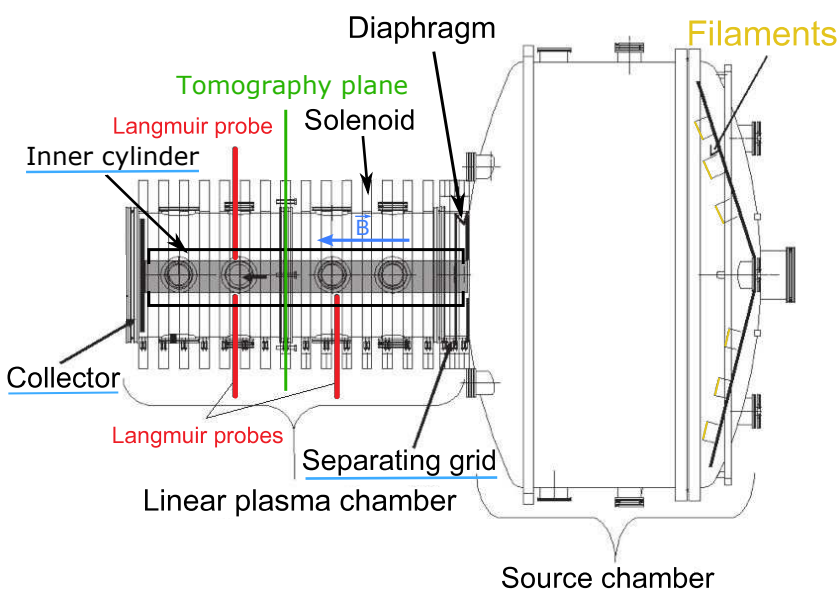

FIG. 1. The Mistral linear plasma device. The blue underlined elements can be individually polarized, controlling the longitudinal (collector and separating grid) and the radial (inner cylinder) boundary conditions of the plasma.

\footnotetext{
a) pierre.david@univ-amu.fr

b) alexandre.escarguel@univ-amu.fr
}

fusion. One of the goals of the tomography diagnostic described here is to study these global rotating modes, and, in particular, address their spatial structure and growth phase. Future works are also aiming at studying turbulent plasma states.

For the experimental conditions considered in this paper, the plasma emission is largely dominated by neutral argon lines ${ }^{5}$. Emission lines are excited from collisions with either primary electrons (coming from the thermoionic discharge), or secondary electrons (coming from the ionisation of argon by primary electrons), with $T_{\mathrm{e}, \text { prim }} \sim 50 \mathrm{eV}, T_{\mathrm{e}, \mathrm{sec}} \sim 3 \mathrm{eV}$, and $n_{\mathrm{e}, \mathrm{prim}} / n_{\mathrm{e}, \mathrm{sec}} \sim 1 \%$. As a result, the plasma emissivity can be written as

$$
E=a . n_{\mathrm{e}, \mathrm{sec}}+b . n_{\mathrm{e}, \mathrm{prim}},
$$

with the coefficients $a$ and $b$ functions of the temperature dependant cross sections for argon excitation. In the experimental conditions of Mistral, the plasma emissivity is dominated by the primary electrons contribution. ${ }^{5}$ Eq. 1 underlines that the plasma emissivity is strongly correlated to the electron density, and is used to compare results of optical diagnostics and of Langmuir probes.

The design of the diagnostic is presented and discussed in Section II. The installation of the diagnostic, and estimation of the noise level and sources are shown in Section III, followed by experimental measurement in Section IV. Finally, the chosen tomography method and its application to a set of experimental data is presented in Section $\mathrm{V}$.

\section{DIAGNOSTIC DESIGN}

The tomography diagnostic of Mistral, shown in Fig. 2 consists of three main parts: the sensors and transimpedance stage, the acquisition board, and the optomechanical system. Two large coplanar windows, on the side and on the top of the linear chamber, allow for an easy optical access to the plasma (shown as the 'tomography plane' in Fig. 1). The optical components being outside the vacuum chamber, there are little mechanical and thermal constraints. 




FIG. 2. Schematic of the tomography diagnostic installed on Mistral.

\section{A. Sensors}

Eight AdvanSiD ASD-RGB4S-P-4x4T, totalling 128 channels, are used to detect incoming photons. The recent technology of Silicone Photomultiplier (SiPM) has been selected over photomultiplier tubes and photodiodes for their high gain $\left(>10^{6}\right.$ electrons per incoming photons), low supply voltage $(<50 \mathrm{~V})$, low price (less than 50 Euro per channel at the time of writing), good time resolution ( $\sim 100$ ns decay time) and good dynamics. Here, the amplification voltage is between 2 and $4 \mathrm{~V}$ beyond the $27 \mathrm{~V}$ breakdown voltage. Their spectral response range is $[350 ; 900] \mathrm{nm}$, peaked at $550 \mathrm{~nm}$ with a photon detection efficiency at maximum overvoltage of $32.5 \%$. Each channel consists in 9340 independent photon counting cells $\left(40 \times 40 \mu^{2}\right.$ each, for a total of $\left.4 \times 4 \mathrm{~mm}^{2}\right)$ connected to a common output producing a summed signal proportional to the number of detected photons. As such, they can be used as photon counting sensors, as well as in a continuous wave regime. Being based on a recent technology, these sensors are still subject to important upgrades every year. The upgrades mainly focus on the sensitivity, the correlated noise (crosstalk and afterpulse), and the fill factor. The crosstalk and afterpulse are the probability of an incoming photon to set a cascade on two adjacent cells, or twice in the same cell, respectively, creating in both cases a additional false positive pulse. The fill factor is the ratio between cells sensitive area over the total area of a channel, thus directly influencing the sensitivity. The three aspects are closely related because a higher fill ratio means a higher sensitivity, but also a higher crosstalk. The sensors used for the work presented in the current paper have a fill factor of $60 \%$. Their gain and combined crosstalk and afterpulse probability vary with the amplification voltage from $0.510^{6}$ to $3.510^{6}$, and 5 to $30 \%$ respectively.



FIG. 3. Picture of one of the two mechanical holder, individually collimating the chords with plastic lenses, and spreading them in a fan shape.

\section{B. Acquisition board}

The electronic acquisition board is a MDU3-GI64X2, developed by AiT-instrument. The board includes the power supply control and the synchronous acquisition of the 128 channels with an integration time down to one microsecond, i.e. an acquisition frequency of $1 \mathrm{MHz}$, on the basis of gated integration method. Its internal memory allows for 4000 acquisitions, before the data need to be transferred to a computer. Its numerical resolution is 12 bits per channel.

\section{Optomechanical system}

Two optical fibre bundles are used to keep the electronic board away from the magnetic field coils and minimise their perturbation. Each end of the 128 fibres facing the plasma is put at the focal point of micro lenses (3.4 $\mathrm{mm}$ in diameter and $9.85 \mathrm{~mm}$ focal length), collimating the line of sight to receive a thin chord of light from the plasma. The diameter of the chord one metre from the lens - upper bound for the distance to the plasmais between 0.9 and $1.2 \mathrm{~cm}$. The micro lenses are mounted on a mechanical holder allowing several chords distributions. In the present case, the chords are set as two fans, one of which is shown in Fig. 3, since fan distributions are known to lessen the effect of noise in the tomographic reconstruction by providing more viewing angles. ${ }^{8}$ On the other side of the bundle, each fibre is placed in front of a channel of the sensor at the distance for which the optical aperture of the fibre covers the whole channel without overflowing on its neighbours.

\section{ALIGNMENT, CONFIGURATION AND CALIBRATION}

Because of the size of the collimation system and the distance to the plasma, a small misalignment can lead to a sizeable displacement of the line of sight. To adjust the collimation system and the chords viewing angle, light has been retro-injected in the 64 optical fibres for each of the two holders, and projected on a wall placed one metre 




FIG. 4. Dark current signal, averaged over 4000 acquisition, of 64 channels versus the temperature of the acquisition board for $+2 \mathrm{~V}$ (top), and $+4 \mathrm{~V}$ (bottom) amplification voltages. Integration time of $10 \mu \mathrm{s}$.

from the lenses. One metre is a compromise between the actual distance to the plasma (roughly $0.5-0.8 \mathrm{~m}$ ), and a distance sufficiently large to easily discriminate between the images of each chord.

The last step before the system can be calibrated and put in use is the characterisation of the acquisition board and the sensors. Because of a high dark current that proved to be a major disturbance at 20 to $30{ }^{\circ} \mathrm{C}$, the cases containing the connection boards with the detectors have been thermally insulated, and are cooled using Peltier units. The heat created by their hot side is dissipated using active liquid coolers. The cooling system can bring the board temperature down to $0{ }^{\circ} \mathrm{C}$. The variation of the dark current signal, with the temperature is shown in Figure 4 on average for 64 channels and two typical amplification voltages. The acquisitions are made with no input light, and for a $10 \mu \mathrm{s}$ integration time. The vertical axes displays the number of events detected on average over 4000 acquisitions. For a temperature less than $7^{\circ} \mathrm{C}$ the dark count becomes very low and does not change much for lower temperatures. In practice, working temperature below $7^{\circ} \mathrm{C}$ is therefore recommended in order to limit the dark count offset that can decrease the available dynamics.

The sensor temperature is monitored by thermocouples to take into account the variation of their sensitivity when the heat produced by the sensors becomes important, i.e. for high light levels or high amplification configurations. Additionally, the breakdown voltage varies with the temperature at $27 \mathrm{mV} /{ }^{\circ} \mathrm{C}$, so keeping a constant gain also requires to monitor the sensor temperature.

Finally, the whole system has been calibrated using a calibrated integrating sphere for measured intensities between $100 \mathrm{pW}$ and a few tens of $\mathrm{nW}$ and for different gains of the sensors. Each channel has a different calibration curve as a result of slight differences in their collimation, breakdown voltages and irregularities between the

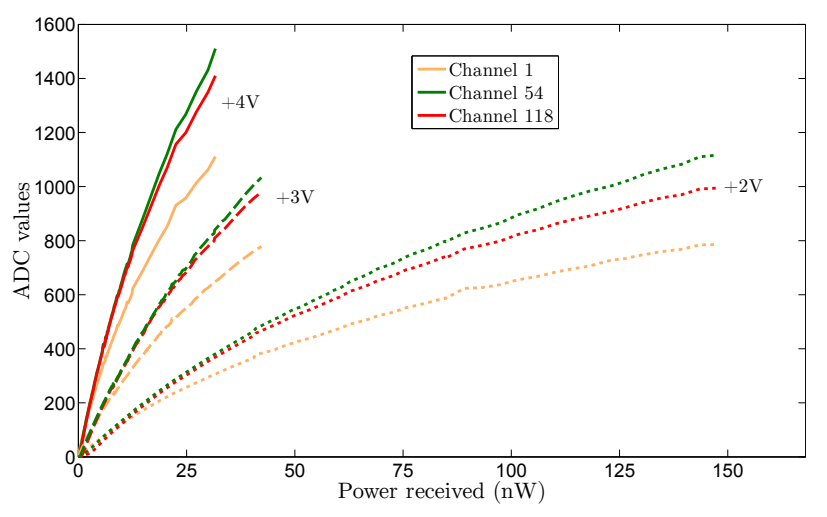

FIG. 5. Calibration curves for three different amplification voltages (dotted, dashed and plain), and for three different channels (orange, red, green).

channels of the electronic board. Moreover, the system is not linear for the whole radiance range. The calibration curves, shown in Fig. 5 for three channels, are fitted with quadratic polynomials which are then used to retrieve the plasma radiance from the measurements.

\section{A. Estimation of the noise}

The noise level of the acquisition board, without the sensors, is slightly above the analog-to-digital convertor noise of 1.5 bits RMS. This is much lower than the noise induced by the sensors. The main noise sources of SiPM are correlated noise (crosstalk and afterpulse), shot noise, and thermal noise. ${ }^{9,10}$ They can be used in photon counting mode for very low light levels, or in continuous wave mode, which is the case for our experimental conditions. In this case the correlated noise only offsets the measured level, but does not add statistical noise, and can be taken into account in the calibration. For a temperature of $7^{\circ} \mathrm{C}$, the remaining shot noise and thermal noise give signal-to-noise ratios between 10 and 100 depending of the integration time, amplification and light level. The better experimental conditions are higher light level and integration time, also allowing a lower amplification, which is consistent with the theoretical formulation as well as numerical simulations for a photon flux between $10^{8}$ and $10^{10}$ photons per second, and a bandwidth of $1 \mathrm{MHz}$ fixed by the sensors.

\section{EXPERIMENTAL MEASUREMENTS}

The measurements presented in this paper have been made on a plasma with the following characteristics: $P_{\text {Argon }}=5 \times 10^{-2} \mathrm{~Pa}, B=16 \mathrm{mT}$, grounded collector and floating separating grid, and the outer cylinder polarised between 20 and $30 \mathrm{~V}$ (see Fig. 1), giving an electron density between $10^{15}$ and $10^{16} \mathrm{~m}^{-3}$. In these conditions, the plasma is very luminous, and exhibits a 


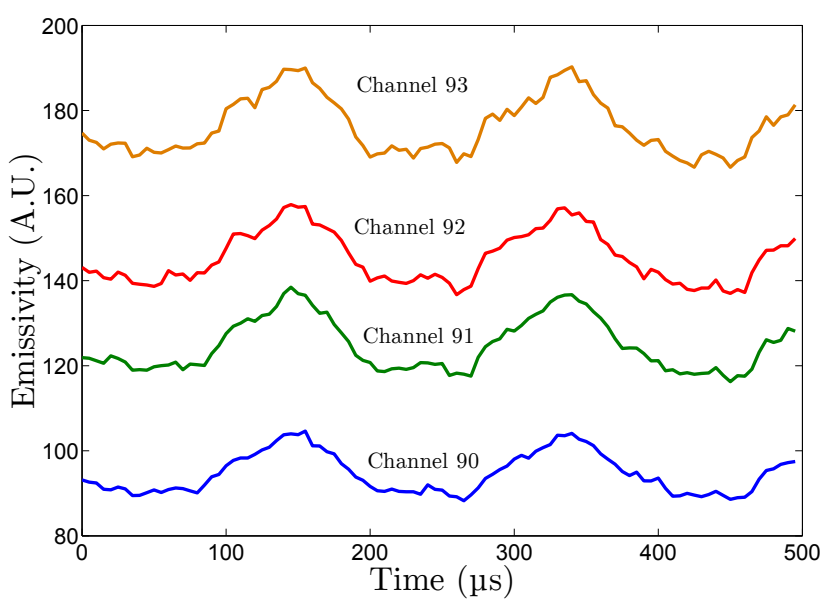

FIG. 6. Temporal evolution of four chords looking at the core plasma from the side, after calibration and conditional averaging.

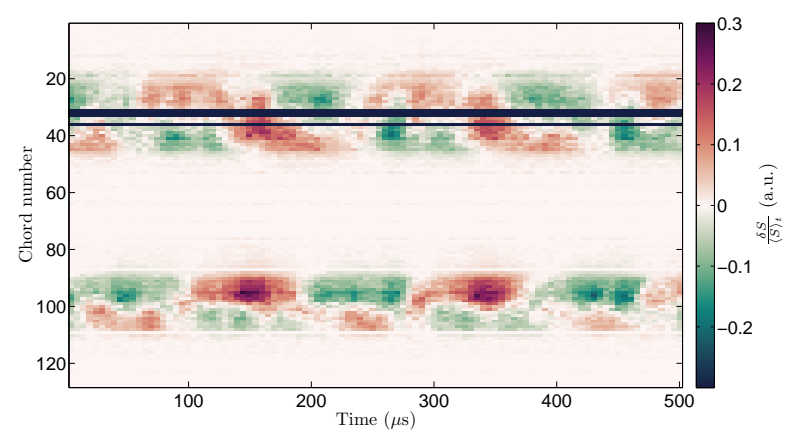

FIG. 7. Variation of the conditionaly averaged intensity, normalised to the averaged intensity for each channel. Channels 1 to 64 look at the plasma from the top, and channels 65 to 128 from the side. Dark lines correspond to non working optical fibers.

rotating arm around the central column, as well as a rigid body rotation of the core plasma, with a period of 200 us. $^{7}$ One hundred acquisitions are performed with a $5 \mu \mathrm{s}$ integration time. Conditional averaging is then performed over 40 acquisition windows. Time evolution of the calibrated and conditional averaged signals for four chords are shown in Fig. 6. In Fig. 7, the variation of the signal after subtraction of the temporal average of each channel is shown, emphasising the oscillation of the plasma. The oscillation can be seen on both the core plasma (chords 25-43 and 90-108), and the edge region. Ideally the signal of the chords seeing the plasma edge should be increased using a higher gain. However, for such experimental conditions, the central chords signal would then saturate the acquisition system. In order to fully exploit the SiPM sensitivity range, additional power supply should be installed. The temporal evolution of the measured signal gives several information of the plasma rotation mode, such as its frequency, intensity, and mode number. Namely, we here have a $m=1$ mode with an

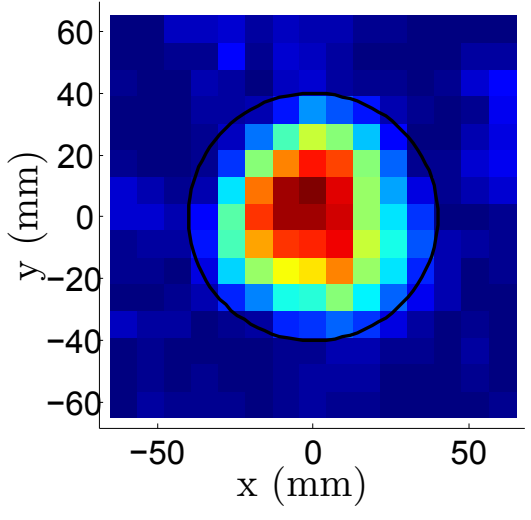

FIG. 8. Temporal average over $500 \mu \mathrm{s}$ of the tomographic inversion of the EMD filtered 128 channels with second order regularisation on a $15 \times 15$ grid.

emissivity variation of about $20 \%$ (positive and negative), rotating at a frequency of $5.3 \mathrm{kHz}$.

\section{NUMERICAL TOMOGRAPHY INVERSION}

Since we are interested not only by the rotating mode general behaviour, but also by their local one (both in time and space), and even non-rotating modes, tomographic inversion the 128 line integrated measurements is used to retrieve the local emissivity. The method used is based on the finite-element scheme described in David et $a l .{ }^{7}$. The plasma cross-section is decomposed into small areas with supposed uniform emissivity, called 'pixels', and the tomography system written as $S=T . E$ with $S=\left(S_{i}\right)$ the signal measured by each channel, $E=\left(E_{j}\right)$ the local emissivity in each pixel, and $T=\left(t_{i j}\right)$ the transfer matrix. In general, the elements $t_{i j}$ of the transfer matrix are the length of the $i^{\text {th }}$ chord in the $j^{\text {th }}$ pixel noted $l_{i j}$, multiplied by a calibration constant (containing the étendue of the chord), noted $C^{\prime}$. However, when lines of sight are collimated by a lens, the full three dimensional calculation brings in an additional factor (see Appendix $\mathrm{A}): t_{i j}=C^{\prime} . l_{i j} . I_{i j}$. The function $I(x)$ is displayed in Fig. 10 for the lenses described in Sec. II C and a $100 \mu \mathrm{m}$ fibre core, and for several distances $\phi$ between the lens and the sensor, to display the effect of imperfect collimation. Three points have to be noted about this function. First, in order to rightfully discretise $I(x)$ into $I_{i j}$, the closest pixels have to be further than a hundred millimetres, otherwise the variations of $I$ can be too important within a pixel. Second, with variation of $\phi$ as little as $0.5 \mathrm{~mm}(<$ $5 \%$ of the focal length), there is an important variation of $I(x)$, with a dependence on the side of the focal point. Finally, $I$ is monotonous for $x>400$ (mm, but the variation is different if we are closer $(\phi<f)$ or further $(\phi>f)$ than the focal point. As a result, each chord of the system has to be precisely collimated and then calibrated to take into account the position of the fibre relative to the lens. 

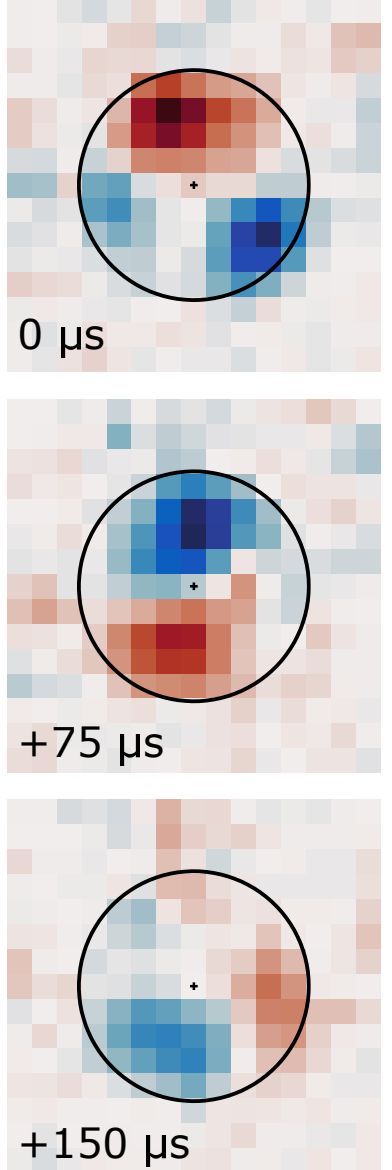
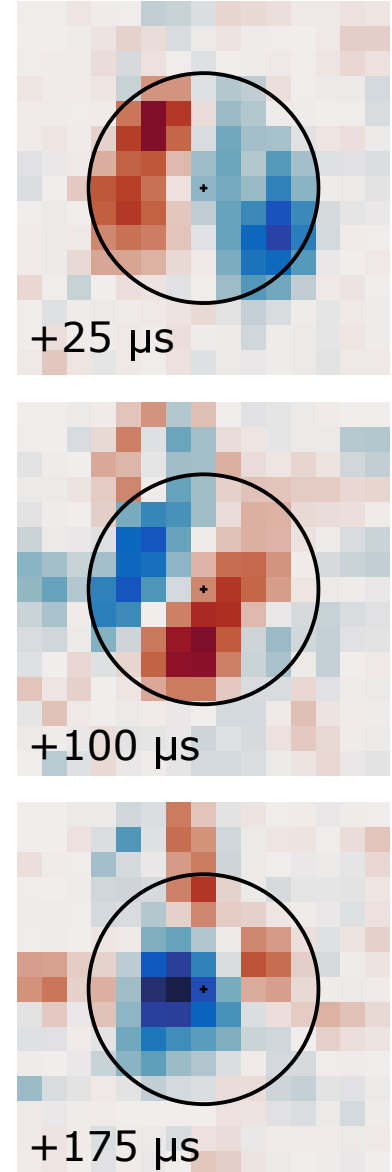
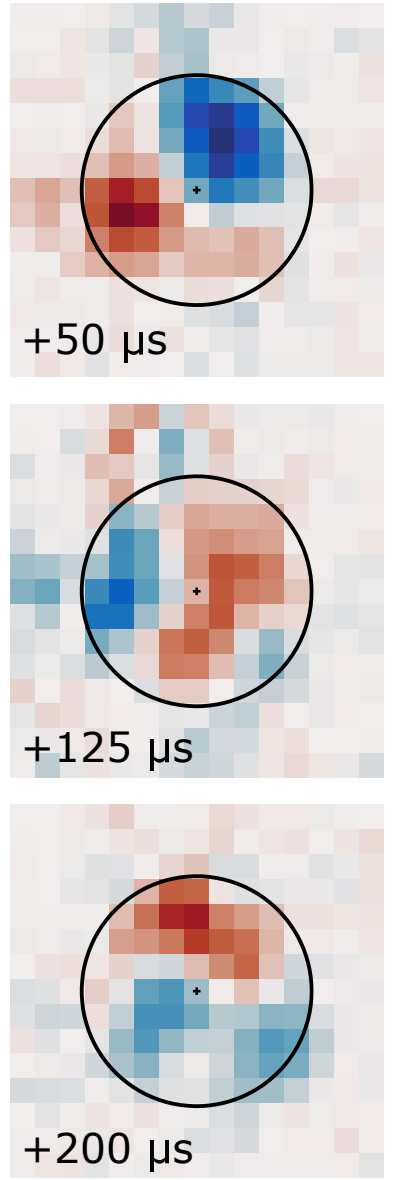

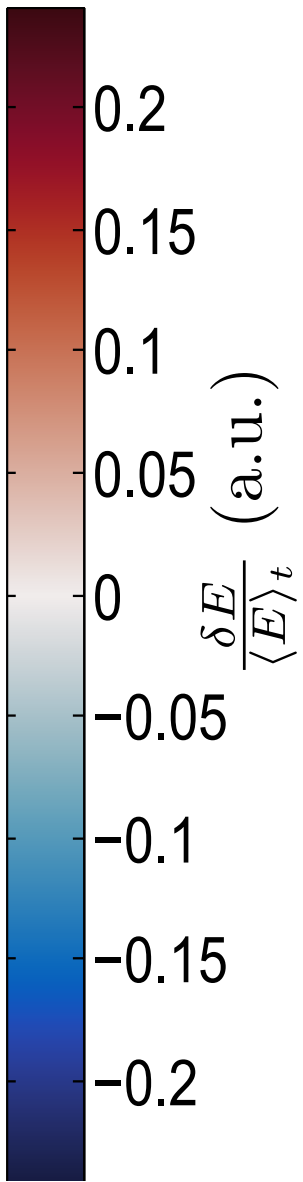

FIG. 9. Tomographic inversion of the EMD filtered 128 channels with second order regularisation on a $15 \times 15$ grid showing the emissiviy variation normalised to the time averaged emissivity at the center (see Fig. 8).

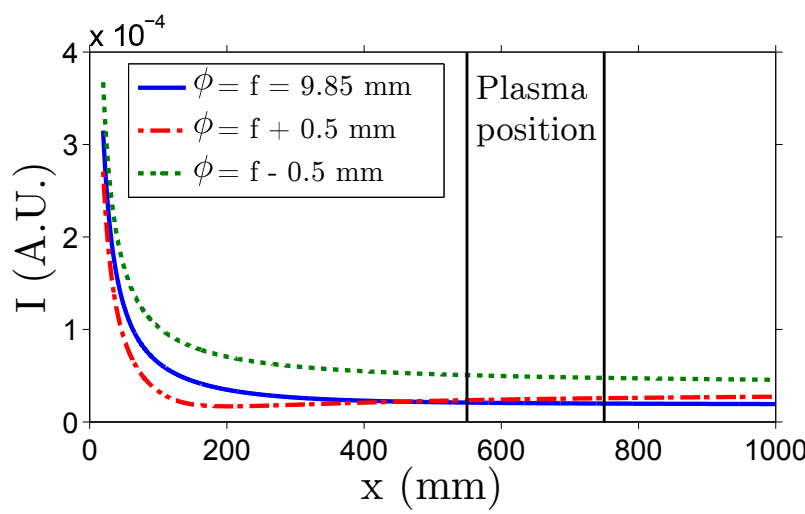

FIG. 10. Variation of the coefficient $I(x)$ weighting the transfer matrix, for different positions of the sensor (here the optical fibre) $\phi$ with respect to the focal plane at $x=f$. Here $f=10.9 \mathrm{~mm}$

The local emissivity in each pixel is obtained by inverting the system $S=T$.E. However, the transfer matrix $T$ is large (128 measurements, and around 200 pixels), ill defined (a small error in the data $E$ will result in large errors in the solutions) and under-defined. As such it has to be regularised. Several regularisation methods were used $^{11}$ : zero, first, and second order derivative, minimisation of the Fisher information, and the SVD decomposition were tested on ghost signals, with the objective of having as few assumptions as possible on the shape of the solution, in order to be able to use the diagnostic on plasma configurations as diverse as possible. The tomography code benchmark has shown consistent results, with less than $15 \%$ of errors in experimentally relevant cases. Best results were more commonly found using the second order derivative regularisation.

In order to lessen the effect of noise without removing too much information from the signal, the Empirical Mode Decomposition (EMD) ${ }^{12}$ has been used to process the experimental measurements (no conditional averaging used in this case). The EMD is based on the concept of instantaneous frequency, using the Hilbert Transform of a signal. The empirical mode decomposition is being increasingly used, and has seen recent upgrades and adaptations to different contexts. Its evolution is easy to follow and to use through several open sources codes, and has already be proven useful in the context of laboratory plasma experiments ${ }^{13}$. The method used here is the CEEMDAN method (for complete ensemble EMD with 
adaptive noise $)^{14}$, available as a Matlab function. Tomographic inversion is then applied to the filtered signal.

Different numbers and shapes of pixels and regularisations methods were tested for robustness assessment. The results were consistent with the code benchmark using ghost images. Tomography inversion with a second order regularisation on a $15 \times 15$ square grid is shown here, each pixel being $9 \mathrm{~mm}$ wide. Fig. 8 displays the temporal average $\langle E\rangle_{t}$ of the tomographic reconstruction from a $500 \mu \mathrm{s}$ acquisition at $200 \mathrm{kHz}$ (one acquisition every $5 \mu \mathrm{s}$. Fig. 9 shows the temporal evolution of the emissivity variation $\delta E$ with respect to its temporal average. $\delta E$ is normalised to the average emissivity of the pixel at the centre. Each image is the actual reconstruction of the section, from a single measurement. The plasma is exhibiting a rotation period of about $200 \mu \mathrm{s}$, consistent with the $195 \mu \mathrm{s}$ measured on average with the trigger probe. The fine spatial structure of the mode can be obtained, and the actual mode number $m=1$ can be explicitly determined.

\section{CONCLUSION}

A visible spectrum tomography diagnostic using 128 SiPM, and an acquisition system for signal integration and acquisition down to the microsecond has been installed on the linear magnetised plasma device Mistral. A numerical tomographic inversion code has been specifically developed for this application, based on a finite volume scheme, and is mostly used with a second order derivative regularisation. Additionally, the exact calculus of the tomography problem was adapted to the chords collimation using lenses.

The whole diagnostic proves to be very promising, and could be installed on smaller plasma devices. Applications of the diagnostic go from the monitoring of the plasma structure during its rotation, including the measurement of the rotating mode number, to the study of the mode growth phase, or the study of burst events.

\section{ACKNOWLEDGMENTS}

This work has been carried out thanks to the support of the A*MIDEX project ( $\mathrm{n}^{\circ}$ ANR-11-IDEX-0001-02) funded by the "Investissements d'Avenir" French Government program, managed by the French National Research Agency (ANR).

\section{Appendix A: Correction factor for collimation with a lens}

Fig. 11 shows three plasma volume elements being collimated to different points. The goal is to determine how much light collected by the lens actually reaches the sensor. The blue element $\left(\mathrm{d} \mathbf{r}_{1}\right)$ of the figure is an example of plasma element from which all emitted light going though the lens is collected by the fibre. However the green and red ( $\mathrm{d} \mathbf{r}_{2}$ and $\mathrm{d} \mathbf{r}_{3}$ respectively) are two different cases in which only part of the light collimated by the lens is collected by the optical fibre, even though the whole lens is within the numerical aperture of the fibre. The rays collimated by the lens have a finite width at the focal plane, and amongst them, only the ones included in the fibre entrance (dotted lines) are actually collected. The ratio of the surfaces defined by the plained lines to the surfaces defined by the dotted lines, both at the position of the optical fibre entrance, gives the full expression for the transfer matrix elements:

$$
t_{i j}=C^{\prime} \cdot l_{i j} \cdot I_{i j}
$$

with $I_{i j}$ the function $I(x)$ taken for $x$ as the distance between the lens and the pixel, and defined by:

$$
I(x)=\left\{\begin{array}{r}
\left(1-\frac{1}{\left.\sqrt{1+\left(\frac{r_{\mathrm{L}}}{x}\right)^{2}}\right)\left(\frac{a^{3}}{6 r_{\mathrm{L}}} x^{3}+\frac{r_{\mathrm{L}} a}{2} x\right)}\right. \\
\text { if } x \in\left[0, \frac{r_{\mathrm{L}}}{r_{\mathrm{F}}}\right] \\
\left(1-\frac{1}{\left.\sqrt{1+\left(\frac{r_{\mathrm{L}}}{x}\right)^{2}}\right)\left(\frac{a^{2}}{2} x^{2}+\frac{r_{\mathrm{L}}^{2}}{6}\right)}\right. \\
\text { if } x \geqslant \frac{r_{\mathrm{L}}}{r_{\mathrm{F}}} f
\end{array}\right.
$$

in which we note $r_{\mathrm{L}}$ and $r_{\mathrm{F}}$ the lens and the fibre core radii, respectively, $f$ the lens focal length, and $a=r_{\mathrm{F}} / f$. The given expressions only work for the optical fibre at the exact focal point.

${ }^{1}$ M. Matsukuma, T. Pierre, A. Escarguel, D. Guyomarc'h, G. Leclert, F. Brochard, E. Gravier, and Y. Kawai, Physics Letters A 314, 163 (2003).

${ }^{2}$ T. Pierre, A. Escarguel, D. Guyomarc'h, R. Barni, and C. Riccardi, Physical Revue Letters 92, 065004 (2004).

${ }^{3}$ S. Jaeger, T. Pierre, and C. Rebont, Physics of Plasmas 16, 022304 (2009), http://dx.doi.org/10.1063/1.3076931.

${ }^{4}$ C. Rebont, N. Claire, T. Pierre, and F. Doveil, Physical Revue Letters 106, 225006 (2011)

${ }^{5}$ A. Escarguel, European Physical Journal D 56, 209 (2010).

${ }^{6}$ B. M. Annaratone, A. Escarguel, T. Lefevre, C. Rebont, N. Claire, and F. Doveil, Physics of Plasmas 18, 032108 (2011).

${ }^{7}$ P. David, A. Escarguel, Y. Camenen, and R. Baude, Physics of Plasmas 23 (2016)

${ }^{8}$ R. Decoste, Review of Scientific Instruments 56, 806 (1985).

${ }^{9}$ P. Finocchiaro, A. Pappalardo, L. Cosentino, M. Belluso, S. Billotta, G. Bonanno, B. Carbone, G. Condorelli, S. D. Mauro, G. Fallica, M. Mazzillo, A. Piazza, D. Sanfilippo, and G. Valvo, IEEE Transactions on Electron Devices 55, 2757 (2008).

${ }^{10}$ G. Adamo, A. Parisi, S. Stivala, A. Tomasino, D. Agro, L. Curcio, G. C. Giaconia, A. Busacca, and G. Fallica, IEEE Journal of Selected Topics in Quantum Electronics 20, 284 (2014).

${ }^{11}$ M. Anton, H. Weisen, M. Dutch, W. Linden, F. Buhlmann, R. Chavan, B. Marletaz, P. Marmillod, and P. Paris, Plasma Physics and Controlled Fusion 38, 1849 (1996).

${ }^{12}$ N. E. Huang, Z. Shen, S. R. Long, M. C. Wu, H. H. Shih, Q. Zheng, N.-C. Yen, C. C. Tung, and H. H. Liu, Proceedings of the Royal Society of London A: Mathematical, Physical and Engineering Sciences 454, 903 (1998). 


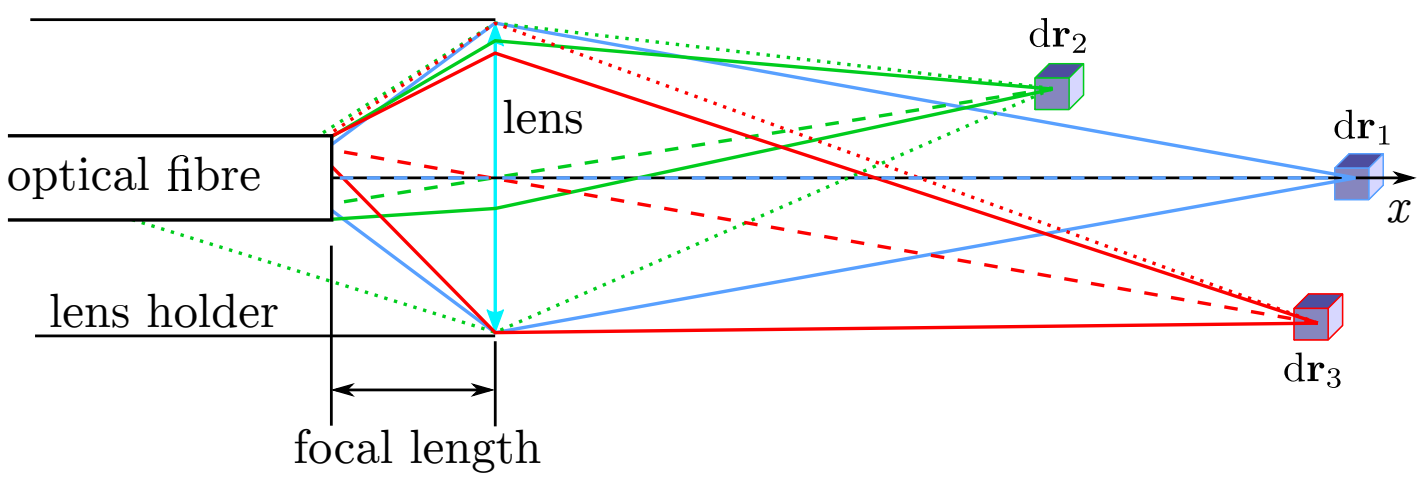

FIG. 11. Light received from three volume elements for a collimation with an optical fibre behind a lens. The plain coloured lines are transmitted by the optical fibre whereas the dotted ones are not. Scales has been freely adapted to beter visualise all the elements.

${ }^{13}$ J. Kurzyna, S. Mazouffre, A. Lazurenko, L. Albarde, G. Bonhomme, K. Makowski, M. Dudeck, and Z. Peradzyński, Physics of Plasmas 12, 123506 (2005), http://dx.doi.org/10.1063/1.2145020.

${ }^{14}$ M. A. Colominas, G. Schlotthauer, and M. E. Torres, Biomedical Signal Processing and Control 14, 19 (2014). 